\title{
The 22nd international symposium on paediatric surgical research
}

\author{
Prem Puri
}

Published online: 22 October 2009

(C) Springer-Verlag 2009

The 22nd International Symposium on Paediatric Surgical Research was held in Genoa, Italy from 16 to 17 September 2009. It was a very successful meeting in which 55 papers related to paediatric surgical research were presented. The Aesculap Prize Session was the highlight of the symposium, with ten excellent papers presented at this session. The Aesculap first prize was awarded to Dr Piotr Hajduk from Dublin for his presentation on "FGF10 Gene Expression is Delayed in the Embryonic Lung Mesenchyme in the Adriamycin Mouse Model". The second prize was awarded to Dr Y. Yu from Hannover for her presentation on "Carbon Dioxide Blocks The Inflamatory Response of Mesothelial Cells and Facilitates Neuroblastoma Cell Migration". The third prize was awarded to Dr S.B. Lulu from Ann Arbor, USA for her presentation on
“Oral Insulin Stimulates Intestinal Epithelial Cell Turnover in Correlation with Insulin Receptor Expression along the Villus-Crypt Axis in a Rat Model of Short Bowel Syndrome".

Professor Paolo De Coppi from UCL Institute of Child Health and Great Ormond Street Hospital for Children, London and Professor Renu Sharma from University of Florida College of Medicine, Florida were the guest speakers at the symposium. Professor De Coppi spoke on "Stem Cells and Paediatric Surgery: What is the future?", and Professor Sharma spoke on "Microecology Intestinal epithelial Barrier and Necrotizing Enterocolitis".

The International Board of Paediatric Surgical Research thanks Professor Vincenzo Jasonni and his team for organising a highly successful meeting in Genoa.
On behalf of the Scientific Committee of the International Board of Paediatric Surgical Research.

P. Puri $(\bowtie)$

Children's Research Centre,

Our Lady's Children's Hospital,

Dublin, Ireland

e-mail: prem.puri@ucd.ie 